\title{
Impact of active screening for methicillin-resistant Staphylococcus aureus (MRSA) and decolonization on MRSA infections, mortality and medical cost: a quasi-experimental study in surgical intensive care unit
}

\author{
Yuarn-Jang Lee ${ }^{1,2}$, Jen-Zon Chen ${ }^{3}$, Hsiu-Chen Lin ${ }^{4,5}$, Hsin-Yi Liu ${ }^{1}$, Shyr-Yi Lin ${ }^{6}$, Hsien-Ho Lin ${ }^{7}$, Chi-Tai Fang ${ }^{7,8 *}$ \\ and Po-Ren Hsueh ${ }^{8, *^{*}}$
}

\begin{abstract}
Introduction: Methicillin-resistant Staphylococcus aureus (MRSA) is a leading pathogen of healthcare-associated infections in intensive care units (ICUs). Prior studies have shown that decolonization of MRSA carriers is an effective method to reduce MRSA infections in ICU patients. However, there is currently a lack of data on its effect on mortality and medical cost.
\end{abstract}

Methods: Using a quasi-experimental, interrupted time-series design with re-introduction of intervention, we evaluated the impact of active screening and decolonization on MRSA infections, mortality and medical costs in the surgical ICU of a university hospital in Taiwan. Regression models were used to adjust for effects of confounding variables.

Results: MRSA infection rate decreased from 3.58 (baseline) to $0.42 \%$ (intervention period) $(P<0.05)$, re-surged to $2.21 \%$ (interruption period) and decreased to $0.18 \%$ (re-introduction of intervention period) $(P<0.05)$. Patients admitted to the surgical ICU during the intervention periods had a lower in-hospital mortality $(13.5 \%(155$ out of 1,147$)$ versus $16.6 \%$ (203 out of 1,226), $P=0.038$ ). After adjusting for effects of confounding variables, the active screening and decolonization program was independently associated with a decrease in in-hospital MRSA infections (adjusted odds ratio: $0.3 ; 95 \%$ Cl: 0.1 to 0.8 ) and 90-day mortality (adjusted hazard ratio: $0.8 ; 95 \%$ Cl: 0.7 to 0.99 ). Cost analysis showed that $\$ 22$ medical costs can be saved for every $\$ 1$ spent on the intervention.

Conclusions: Active screening for MRSA and decolonization in ICU settings is associated with a decrease in MRSA infections, mortality and medical cost.

\section{Introduction}

Methicillin-resistant Staphylococcus aureus (MRSA), first reported from England in 1961, is a leading pathogen of nosocomial infections in intensive care units (ICUs) $[1,2]$. In recent years, reduced susceptibility to vancomycin has made MRSA more difficult to treat than before [3,4]. Patients who have healthcare-associated

\footnotetext{
* Correspondence: fangct@ntu.edu.tw; hsporen@ntu.edu.tw

'Institute of Epidemiology and Preventive Medicine, College of Public Health,

National Taiwan University, 17 Xu-Zhou Road, Taipei 10002, Taiwan

${ }^{8}$ Department of Internal Medicine, National Taiwan University Hospital, 7

Chun-Shan South Road, Taipei 10002, Taiwan

Full list of author information is available at the end of the article
}

MRSA (HA-MRSA) infections have increased mortality risk and prolonged hospital stay, resulting in increased medical costs, compared with patients who do not have HA-MRSA infections [5].

A significant proportion of MRSA infections are endogenous and are caused by the same strain that colonizes the nasal mucosa [6,7]. Observational studies [8-12] and the REDUCE MRSA trial [13] have consistently shown that decolonization of ICU patients, using intranasal mupirocin and chlorhexidine body-washing, can reduce MRSA infection rates. Decolonization directly reduces endogenous infections in carriers, and indirectly 
reduces exogenous infections in non-carriers. Nevertheless, whether the ultimate goals of infection control, that is, the reduction of medical cost and mortality, can be achieved by these sorts of interventions remains unsettled, as previous studies did not look for these outcomes $[14,15]$.

In Taiwan, MRSA was first reported in the 1980s [16]. The proportion of MRSA among all S. aureus isolates that cause infections in ICUs has increased to approximately $80 \%[16,17]$. In our hospital, MRSA infection rates in the ICU remained high, despite efforts on contact isolation and decolonization of patients with clinical MRSA infections. To control the problem, a routine active MRSA screening and decolonization program was implemented in the surgical ICU (SICU), which led to a rapid drop in MRSA infection rate. The program was temporarily suspended between May 2008 and August 2009, owing to a lack of financial support, followed by a resurge in MRSA infection rate. The program was then restarted in September 2009, and the MRSA infection rate rapidly decreased again.

Using a quasi-experimental study design, we sought to evaluate the impact of active screening and decolonization of ICU patients, including both direct and indirect protective effect, on the incidence of MRSA infections, mortality and medical costs.

\section{Methods}

\section{Setting}

This study was conducted in the SICU of Taipei Medical University Hospital (TMUH), a tertiary care, universityaffiliated teaching hospital in northern Taiwan. TMUH has a 702-bed capacity. The SICU has 18 beds (all are single bed rooms).

\section{Ethical statement}

The institutional review board (IRB) of TMUH approved the study protocol (protocol number: TMUH-05-11-04). The IRB approved the waiver of informed consent (see Additional file 1).

\section{Study design}

This was a quasi-experimental, interrupted time-series study [18]. Regression models were used to adjust for the effects of confounding variables, including hospital-level infection-control practices (hand hygiene and bundle care) and patient-level risk factors (invasive procedures and severity of underlying diseases). Data on MRSA infection rate, mortality and medical cost were retrospectively obtained from computer databases.

The study period was divided into four stages. In period one (baseline, between January and September 2007), contact precautions, eradication and environmental disinfection at discharge were performed only for those patients with positive clinical cultures for MRSA. In period two (intervention period), routine active screening and decolonization (supported by a research grant from the hospital) was initiated and lasted between October 2007 and April 2008. The intervention was halted in period three (interruption period, between May 2008 and August 2009) owing to a lack of research grants. After a resurgence in the SICU MRSA infection rates during period three prompted the hospital leadership to provide financial support for the active screening and decolonization program, the intervention was resumed in period four (reintroduction period, between September 2009 and September 2010) (Figure 1).

We compared the HA-MRSA infection rates and mortality rates [5] of all patients admitted during the intervention periods (period two and period four) to those of patients admitted during the non-intervention periods (period one and period three), after adjusting for effects of other variables that may influence the outcomes.

\section{Study interventions}

The intervention consisted of active surveillance cultures that were immediately taken from the anterior nares of the patients at the time of admission to the SICU to identify asymptomatic MRSA carriers. Extra-nasal cultures were not obtained. Before culture results became available, patients were put in a single-bed room and standard precautions were applied. If nasal swab cultures were positive, MRSA was eradicated from the nares by the application of mupirocin ointment (GlaxoSmithKline, GSK, Crawley, United Kingdom) three times per day for five days, and from the skin by the application of $4 \%$ chlorhexidine gluconate (Panion \& BF Biotech Incorporation, Taoyuan, Taiwan) once per day for five days; contact precautions were also taken. Patients were screened for MRSA carriage only at their admission, rather than regularly during their stay in ICU.

\section{Microbiological procedures}

During the intervention periods (period two and period four), the nasal swab was plated on blood agar plates, which were incubated at $35^{\circ} \mathrm{C}$ with $5 \% \mathrm{CO}_{2}$ in ambient air, and were checked for the presence of $S$. aureus after 16 to 18 hours of incubation. To shorten the time interval from culture to reporting MRSA, suspected S. aureus isolates were tested for the presence of coagulase using BD BBL Coagulase Plasma Rabbit with EDTA (BD, Franklin Lakes, $\mathrm{NJ}$, USA) $\left(35^{\circ} \mathrm{C}\right.$ overnight), and plated on oxacillin screen agar plates containing $6 \mu \mathrm{g} / \mathrm{ml}$ oxacillin and $4 \% \mathrm{NaCl}$ (BD, Franklin Lakes, NJ, USA) at $35^{\circ} \mathrm{C}$ for 24 hours to test for oxacillin resistance.

\section{Hospital-level infection control practices}

Hand hygiene practice continued to improve during the study period. The increase in hand washing was measured by the amounts of alcoholic disinfectant used for 


\section{Baseline Intervention Interruption Re-introduction \\ $\begin{array}{llll}\text { Period } 1 & \text { Period } 2 & \text { Period } 3 & \text { Period } 4\end{array}$

$\begin{aligned} & \text { Jan 1,2007- } \\ & \text { Sep 30, 2007 }\end{aligned}>\begin{aligned} & \text { Oct 1,2007- } \\ & \text { Apr 30,2008 }\end{aligned}>\begin{aligned} & \text { May 1,2008- } \\ & \text { Aug 31,2009 }\end{aligned}>>\begin{aligned} & \text { Sep 1,2009- } \\ & \text { Sep 30,2010 }\end{aligned}$ \\ Non-intervention Periods

Non-intervention period:
- Decolonization only for
MRSA clinical patients.
- Contact isolation \\ Intervention Periods \\ Intervention period : \\ - Active surveillance and decolonization treatment for MRSA carriers at SICU admission \\ - Contact isolation}

Figure 1 Study design: period one (baseline), period two (intervention period), period three (interruption period) and period four (re-introduction of intervention period). MRSA: methicillin-resistant Staphylococcus aureus; SICU: surgical intensive care unit.

hand washing per 1,000 patient days (15.7 liters in period 1, 16.4 liters in period 2, 21.9 liters in period 3 and 23.4 liters in period 4), based on hospital administrative data.

There was no change in antibiotic prescribing patterns or overall sepsis management during the study period. No cohorting was used. Ventilator-associated pneumonia (VAP) bundle care, including head elevation, daily interruption of sedation for assessing extubation, and daily $0.2 \%$ chlorhexidine mouth cleansing, started in August 2009.

\section{Individual patient-level risk factors}

Data on the severity of underlying diseases, including Acute Physiology and Chronic Health Evaluation II (APACHE II) score upon admission to the ICU, length of hospital stay before ICU admission and invasive procedures, were routinely recorded. We retrospectively obtained patient-level data from the hospital's computer system database. The APACHE II scores were divided into two groups (low and high), using a cutoff point of 15 [19].

\section{Outcome ascertainment}

In the study hospital, infection control nurses routinely review all hospitalizations for all types of healthcareassociated infections (HAIs) using the Centers for Disease Control and Protection (CDC) surveillance definitions $[20,21]$. The surveillance practice for detecting the HAIs remained the same throughout the study period. Data on HA-MRSA cases that occurred during the study period were retrospectively obtained from the routine surveillance records. The SICU HA-MRSA infection rate was defined as the number of HA-MRSA infections per 1,000 patient SICU days. In-hospital HA-MRSA infection rate was defined as the number of HA-MRSA infections per 1,000 patient days from the time of SICU admission to discharge.

The in-hospital mortality of the patients was ascertained using hospital medical records and death certificates. The 90-day mortality (from SICU admission, including deaths occurring after discharge) of the patients was ascertained using the National Death Registry [5], updated to the end of 2010. To protect the privacy of patients, personal identification numbers were scrambled and anonymized before database linkage.

\section{Medical cost}

Data on length of hospital stay and medical costs were obtained from the National Health Insurance Claims database [5] of the study hospital. The list of medical costs included the costs for physician care, accommodations, nursing care, pharmacy services, laboratory procedures, operations, rehabilitation programs, medications and anesthesia.

\section{Cost of intervention}

All costs were calculated using an exchange rate of US\$1 = NT\$30. The costs for active screening included the cost of the materials for microbiological procedures (US\$5 (NT\$150) per ICU patient) and the cost of nursing personnel (US\$6.7 (NT\$200)/hour $\times 5$ minutes per ICU patient) and laboratory technicians (US\$5 (NT\$150)/ hour $\times 10$ minutes per patient). The cost for decolonization included the cost of mupirocin (1 tube at US\$1.7 
(NT\$51), per MRSA carrier) and chlorhexidine $(20 \mathrm{ml}$ at US\$0.19 (NT\$5.8), per MRSA carrier).

The costs for contact isolation included the costs of gloves (US\$0.05 (NT\$1.6) per unit), surgical masks (US\$0.04 (NT\$1.28) per unit), aprons (cleansing and disinfection) (US\$0.003 (NT\$0.08) per unit) and alcohol disinfectants for hand washing $(10 \mathrm{ml}$ at US\$0.12 (NT\$3.64), after each contact). There were, on average, fifty contacts per day (including forty nursing contacts, two physician contacts, four respiratory therapist contacts, two nursing specialist contacts, and two paramedic contacts), for a total of fourteen days of isolation for each MRSA carrier. We assumed a $100 \%$ adherence to contact precautions, with gloves, mask and apron changed after each contact, as well as hand washing.

\section{Statistical analyses}

All analyses were conducted using SAS version 9.2 (SAS Institute, Cary, NC, USA). Risk factors for MRSA infections or mortality were analyzed using logistic regression or Cox regression. For multivariate analyses, all potential risk factors were included in the maximum model. We used stepwise regression procedures to identify independent risk factors. All tests were two-tailed, and $P<0.05$ was considered to be statistically significant.

\section{Number of MRSA infections averted}

We estimated the number of MRSA infections that would have occurred in the absence of the intervention program using the following formula:

Total patient days in period two $\times$ (observed MRSA infection rate in period two/adjusted hazard ratio of intervention) + Total patient days in period four $\times$ (observed
MRSA infection rate in period four/adjusted hazard ratio of intervention).

The number of MRSA infection cases averted by the intervention program was estimated using the predicted numbers (in the absence of the program) in Periods 2 and 4 minus the observed number in Periods 2 and 4.

\section{Cost-saving analysis}

The cost saved by the intervention was estimated by multiplying the number of averted MRSA infections with the median excess total hospitalization for each MRSA infection case. We estimated the cost saved for every dollar spent on active screening and decolonization program by dividing the cost saved for the averted MRSA infections by the cost of implementing the intervention.

\section{Results}

\section{Baseline characteristics}

A total of 2,373 patients were admitted to the SICU during the study period. Table 1 shows the baseline characteristics of the patients admitted during the different periods.

\section{Active surveillance cultures}

Of the 314 patients admitted in period 2, 213 (67.8\%) received active screening for MRSA. The remaining 101 patients did not receive such surveillance cultures because of mortality soon after admission or rapid transfer to other wards. Similarly, of the 833 patients admitted in period 4, 538 (64.5\%) received surveillance cultures. The average ICU-admission-to-culture-reporting time was 2.5 days. Among surveillance cultures, the positive rate

Table 1 Baseline characteristics of the 2,373 patients admitted to the surgical ICU

\begin{tabular}{|c|c|c|c|c|c|c|}
\hline Variable & $\begin{array}{l}\text { Period } 1 \\
\text { (non-intervention) } \\
(n=327)\end{array}$ & $\begin{array}{l}\text { Period } 2 \\
\text { (intervention) } \\
(n=314)\end{array}$ & $P$ value & $\begin{array}{l}\text { Period } 3 \\
\text { (non-intervention) } \\
(n=899)\end{array}$ & $\begin{array}{l}\text { Period } 4 \\
\text { (intervention) } \\
(n=833)\end{array}$ & $P$ value \\
\hline Age (mean $\pm S D$ ) & $62 \pm 18$ & $65 \pm 17$ & 0.060 & $61 \pm 18$ & $62 \pm 18$ & 0.234 \\
\hline Age >65 (years) & $163(49.8)$ & $177(56.4)$ & 0.098 & $424(52.9)$ & $408(51.1)$ & 0.450 \\
\hline Male & $204(62.4)$ & $189(60.1)$ & 0.569 & $543(60.4)$ & $481(57.7)$ & 0.261 \\
\hline Pre-ICU LOS (mean \pm SD) & $4.1 \pm 7.7$ & $5.5 \pm 12.4$ & 0.104 & $3.5 \pm 7.4$ & $3.2 \pm 6.7$ & 0.310 \\
\hline APACHE II (median) & 16 & 15 & 0.368 & 10 & 10 & 0.263 \\
\hline APACHE $\|>15$ & $182(55.7)$ & $157(50)$ & 0.151 & $271(30.2)$ & $260(31.3)$ & 0.630 \\
\hline Endotracheal intubation & $58(17.7)$ & $50(15.9)$ & 0.540 & $124(13.7)$ & $102(12.2)$ & 0.340 \\
\hline Operation & $210(64.2)$ & $229(72.9)$ & 0.018 & $680(75.6)$ & $594(71.3)$ & 0.036 \\
\hline CVC catheter & $164(50.1)$ & $169(53.8)$ & 0.353 & $451(50.1)$ & $395(47.4)$ & 0.253 \\
\hline Foley catheter & $128(39.1)$ & $104(33.1)$ & 0.113 & 300 (33.3) & 305 (36.6) & 0.157 \\
\hline Double lumen catheter & $11(3.4)$ & $11(3.5)$ & 0.923 & $36(4)$ & $27(3.2)$ & 0.397 \\
\hline
\end{tabular}

Data are number (\%) unless otherwise specified. APACHE II: Acute Physiology and Chronic Health Evaluation II; CVC: Central venous catheter; Pre-ICU LOS: before ICU length of hospital stay (days). 
in periods 2 and 4 was $11.3 \%$ (24 out of 213 ) and $6.1 \%$ (33 out of 538), respectively $(P=0.028)$.

\section{Healthcare-associated MRSA infection rates in the surgical ICU}

Twenty-three patients developed MRSA infections (including eleven bloodstream infections, eight respiratory tract infections, two urinary tract infections, one cardiovascular system infection and one eye, ear, nose, throat or mouth infection) during their stay in the SICU in non-intervention periods, compared with two patients (two respiratory tract infections) during the intervention periods (Table 2). After the start of intervention, the monthly MRSA infection rate in the SICU rapidly dropped to zero (Figure 2) (overall MRSA infection rate: $3.58 \%$ (period 1) versus $0.42 \%$ (period 2 ), $P<0.05$ ). After the suspension of the program in May 2008, the monthly MRSA infection rates in the SICU rapidly resurged and rose to 12\%o in August 2009 (Figure 2), despite an improved hand hygiene practice from 16.44 to 21.87 liters per 1,000 patient days. After re-introduction of the intervention program in September 2009, the monthly MRSA infection rate rapidly dropped to zero again (Figure 2) (overall MRSA infection rate: 2.21\% (period 3 ) versus $0.18 \%$ (period 4$), P<0.05$ ).

Effect of intervention on in-hospital MRSA infections Univariate logistic regression analysis showed that the active screening and decolonization program was a significant protective factor for in-hospital MRSA infections (Table 3). Multiple logistic regression analysis showed that the intervention was an independent protective factor (adjusted odds ratio (OR): 0.3 ; 95\% CI: 0.1 to 0.8 ) (Table 3).

Univariate Cox regression analysis showed that the active screening and decolonization program was a significant protective factor for MRSA infections (Table 4). Multiple Cox regression analysis showed that the intervention was an independent protective factor (adjusted hazard ratio (HR): 0.3 ; 95\% CI: 0.1 to 0.7 ) (Table 4 ).

\section{In-hospital mortality}

The in-hospital mortality rate of patients admitted to the SICU was $19.3 \%$ (63 out of 327 ) in period 1, which decreased to $13.7 \%$ (43 out of 314 ) in period 2 (intervention period), increased again to $15.6 \%$ (140 out of 899 ) in period 3 (interruption period) and decreased again to 13.4\% (112 out of 833) in period 4 (re-introduction period). Patients admitted to the SICU during the intervention periods had a significantly lower in-hospital mortality than those admitted during the non-intervention periods (13.5\% (155 out of 1,147) versus 16.6\% (203 out of $1,226) ; P=0.038$; chi-square test).

\section{Effect of intervention on 90-day mortality}

Table 5 shows the results of Cox regression analysis for risk factors of mortality within 90 days. Multiple Cox regression analysis showed that the intervention was an independent protective factor against mortality

Table 2 Healthcare-associated MRSA infection rates

\begin{tabular}{|c|c|c|c|c|}
\hline Variable & $\begin{array}{l}\text { Period } 1 \\
\text { Non-intervention } \\
(\mathrm{n}=327)\end{array}$ & $\begin{array}{l}\text { Period } 2 \\
\text { Intervention } \\
(n=314)\end{array}$ & $\begin{array}{l}\text { Period } 3 \\
\text { Non-intervention } \\
(\mathrm{n}=899)\end{array}$ & $\begin{array}{l}\text { Period } 4 \\
\text { Intervention } \\
(n=833)\end{array}$ \\
\hline Total patient days (SICU) & 2,792 & 2,371 & 5,875 & 5,434 \\
\hline Total patient days (in-hospital) & 7,028 & 6,817 & 17,387 & 16,523 \\
\hline$\%$ of patients screened at ICU admission & - & $67.8 \%(213 / 314)$ & - & $64.5 \%(538 / 833)$ \\
\hline MRSA positive rate of surveillance culture & - & $11.3 \%(24 / 213)$ & - & $6.1 \%(33 / 538)$ \\
\hline MRSA infection rate (\%o) (SICU) & 3.58 & 0.42 & 2.21 & 0.18 \\
\hline SICU MRSA infection (number) & 10 & $1^{\mathrm{a}}$ & 13 & $1^{\mathrm{a}}$ \\
\hline BSI infection & 6 & 0 & 5 & 0 \\
\hline RTI infection & 3 & 1 & 5 & 1 \\
\hline UTI infection & 0 & 0 & 2 & 0 \\
\hline SSI infection & 0 & 0 & 0 & 0 \\
\hline Other infection sites (CVSI, EENTI) & 1 & 0 & 1 & 0 \\
\hline MRSA infection rate (\%o) (in-hospital) & 1.42 & 0.29 & 0.75 & 0.24 \\
\hline In-hospital MRSA infection (number) & 10 & $2^{\mathrm{b}}$ & 13 & $4^{\mathrm{b}}$ \\
\hline
\end{tabular}

${ }^{a}$ Both cases had negative surveillance culture at the time of SICU admission. ${ }^{b}$ Including the two SICU MRSA infection cases. Of the four MRSA infection cases that occurred after transfer to general wards, three cases had negative surveillance culture at the time of SICU admission, and the other had positive surveillance culture at the time of SICU admission. BSI: Bloodstream infection; CVSI: Cardiovascular system infection; EENTI: eye, ear, nose, throat, or mouth infection; MRSA: Methicillin-resistant Staphylococcus aureus; RTI: Respiratory tract infection; SICU: Surgical intensive care unit; SSI: Surgical site infection; UTI: Urinary tract infection. 


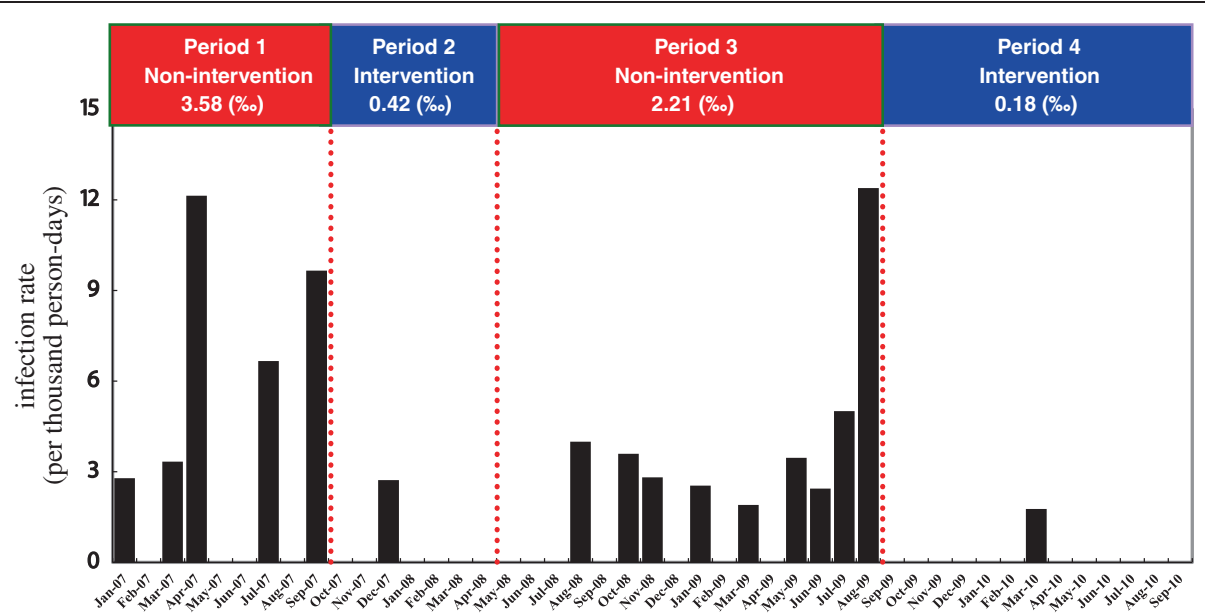

Figure 2 Monthly incidence of healthcare-associated methicillin-resistant Staphylococcus aureus infections during non-intervention periods (period one and period three) and intervention periods (period two and period four) in the surgical intensive care unit.

(adjusted HR: 0.8; 95\% CI: 0.7 to 0.99$)$, after adjusting for the effects of the other variables (Table 5).

\section{Excess length of hospital stay}

Mean length of hospital stay was significantly higher for patients with MRSA infections than for those without MRSA infections (in SICU: 40.6 days versus 6.6 days; total hospitalization: 75.4 days versus 23.3 days; both

Table 3 Risk factors for in-hospital MRSA infections

\begin{tabular}{|c|c|c|c|c|}
\hline \multicolumn{3}{|c|}{ Univariate logistic regression analysis } & \multicolumn{2}{|c|}{$\begin{array}{l}\text { Multiple logistic } \\
\text { regression analysis }\end{array}$} \\
\hline Variable & OR $(95 \% \mathrm{Cl})$ & $P$ value & $\begin{array}{l}\text { Adjusted OR } \\
(95 \% \mathrm{Cl})\end{array}$ & $P$ value \\
\hline Age >65 (years) & $2.1(0.9-4.7)$ & 0.077 & & \\
\hline Sex & $1.0(0.5-2.1)$ & 0.961 & & \\
\hline Pre-ICU LOS & $1.0(1.0-1.1)$ & 0.148 & & \\
\hline Operation & $1.4(0.5-3.3)$ & 0.513 & & \\
\hline CVC catheter & $1.7(0.8-3.8)$ & 0.166 & & \\
\hline Foley catheter & $3.2(1.5-7.0)$ & 0.004 & & \\
\hline $\begin{array}{l}\text { Double lumen } \\
\text { catheter }\end{array}$ & $3.5(1.0-12.0)$ & 0.042 & & \\
\hline APACHE $\|>15$ & $3.0(1.4-6.5)$ & 0.007 & $2.6(1.1-6.0)$ & 0.025 \\
\hline $\begin{array}{l}\text { Endotracheal } \\
\text { intubation }\end{array}$ & $4.3(2.0-9.4)$ & 0.0001 & $3.5(1.6-8.0)$ & 0.002 \\
\hline Antibiotic use & $1.4(0.6-3.1)$ & 0.469 & & \\
\hline Hand hygiene $e^{a, b}$ & $0.9(0.8-1.0)$ & 0.029 & & \\
\hline VAP bundle & $0.3(0.1-0.8)$ & 0.021 & & \\
\hline Intervention & $0.3(0.1-0.8)$ & 0.009 & $0.3(0.1-0.8)$ & 0.015 \\
\hline
\end{tabular}

${ }^{a}$ Hand hygiene: the amount of alcoholic disinfectant (liter) used for hand washing per 1,000 patient days during a period. ${ }^{b} P=0.569$ for the interaction between intervention and hand hygiene. APACHE II: Acute Physiology and Chronic Health Evaluation II; CVC: central venous catheter; ICU LOS: ICU length of hospital stay; OR: odds ratio; MRSA: methicillin-resistant Staphylococcus aureus; VAP: ventilator-associated pneumonia.
$P<0.001$ ), with an excess of 34.0 days (in SICU) and 52.1 days (total hospitalization) (Table 6).

\section{Excess cost for patients with MRSA infections}

The mean hospital cost was significantly higher for patients with MRSA infections than for those without MRSA infections (in SICU: US\$25,466 versus US\$6,612; total hospitalization: US $\$ 31,815$ versus US $\$ 8,505$; both

Table 4 Risk factors for time to in-hospital MRSA infections

\begin{tabular}{|c|c|c|c|c|}
\hline \multicolumn{3}{|c|}{ Univariate Cox regression analysis } & \multicolumn{2}{|c|}{$\begin{array}{l}\text { Multiple Cox } \\
\text { regression analysis }\end{array}$} \\
\hline Variable & HR $(95 \% \mathrm{Cl})$ & $P$ value & $\begin{array}{l}\text { Adjusted HR } \\
(95 \% \mathrm{Cl})\end{array}$ & $P$ value \\
\hline Age $>65$ (years) & $1.5(0.7-3.4)$ & 0.307 & & \\
\hline Sex & $1.0(0.5-2.2)$ & 0.972 & & \\
\hline pre-ICU LOS & $1.0(1.0-1.0)$ & 0.680 & & \\
\hline Operation & $1.1(0.4-2.6)$ & 0.913 & & \\
\hline CVC catheter & $1.1(0.5-2.4)$ & 0.858 & & \\
\hline Foley catheter & $1.0(0.4-2.3)$ & 0.994 & & \\
\hline $\begin{array}{l}\text { Double lumen } \\
\text { catheter }\end{array}$ & $1.5(0.4-5.2)$ & 0.505 & & \\
\hline APACHE $\|>15$ & $2.1(1.0-4.6)$ & 0.066 & $2.3(1.0-5.3)$ & 0.037 \\
\hline $\begin{array}{l}\text { Endotracheal } \\
\text { intubation }\end{array}$ & $1.7(0.8-3.9)$ & 0.177 & & \\
\hline Antibiotic use & $1.2(0.5-2.8)$ & 0.657 & & \\
\hline Hand hygiene ${ }^{a, b}$ & $0.9(0.8-1.0)$ & 0.051 & & \\
\hline VAP bundle & $0.3(0.1-0.9)$ & 0.025 & & \\
\hline Intervention & $0.3(0.1-0.7)$ & 0.010 & $0.3(0.1-0.7)$ & 0.006 \\
\hline
\end{tabular}

${ }^{\mathrm{a}}$ Hand hygiene: the amount of alcoholic disinfectant (liter) used for hand washing per 1,000 patient days during a period. ${ }^{\mathrm{b}} P=0.597$ for the interaction between intervention and hand hygiene. APACHE II: Acute Physiology and Chronic Health Evaluation II; CVC: central venous catheter; HR: hazard ratio; ICU LOS: ICU length of hospital stay; MRSA: methicillin-resistant Staphylococcus aureus; VAP: ventilator-associated pneumonia. 
Table 5 Risk factors for 90-day mortality

\begin{tabular}{|c|c|c|c|c|}
\hline \multicolumn{3}{|c|}{ Univariate Cox regression analysis } & \multicolumn{2}{|c|}{$\begin{array}{l}\text { Multiple Cox } \\
\text { regression analysis }\end{array}$} \\
\hline Variable & HR $(95 \% \mathrm{Cl})$ & $P$ value & $\begin{array}{l}\text { Adjusted HR } \\
(95 \% \mathrm{Cl})\end{array}$ & $P$ value \\
\hline Age $>65$ (years) & $2.4(1.9-2.8)$ & $<0.0001$ & $1.5(1.2-1.8)$ & 0.0002 \\
\hline Sex & $1.0(0.8-1.1)$ & 0.730 & & \\
\hline pre-ICU LOS & $1.0(1.0-1.0)$ & $<0.0001$ & $1.0(0.99-1.0)$ & 0.421 \\
\hline Operation & $0.4(0.3-0.5)$ & $<0.0001$ & $0.3(0.3-0.4)$ & $<0.0001$ \\
\hline CVC catheter & $1.6(1.3-1.9)$ & $<0.0001$ & $1.5(1.2-1.9)$ & 0.0002 \\
\hline Foley catheter & $2.6(2.2-3.2)$ & $<0.0001$ & $1.4(1.2-1.7)$ & 0.0007 \\
\hline $\begin{array}{l}\text { Double lumen } \\
\text { catheter }\end{array}$ & $4.3(3.2-5.7)$ & $<0.0001$ & $1.7(1.2-2.3)$ & 0.001 \\
\hline APACHE $\|>15$ & $3.8(3.1-4.5)$ & $<0.0001$ & $2.5(2.0-3.0)$ & $<0.0001$ \\
\hline $\begin{array}{l}\text { Endotracheal } \\
\text { intubation }\end{array}$ & $3.8(3.1-4.5)$ & $<0.0001$ & $1.5(1.2-1.9)$ & 0.0003 \\
\hline Hand hygiene $e^{a, b}$ & $0.95(0.9-0.98)$ & 0.002 & $1.0(0.9-1.0)$ & 0.617 \\
\hline Intervention & $0.9(0.7-1.1)$ & 0.219 & $0.8(0.7-0.99)$ & 0.048 \\
\hline
\end{tabular}

${ }^{\mathrm{a}}$ Hand hygiene: the amount of alcoholic disinfectant (liter) used for hand washing per 1,000 patient days during a period. ${ }^{\mathrm{b}} P=0.16$ for the interaction between intervention and hand hygiene. APACHE II: Acute Physiology and Chronic Health Evaluation II; CVC: central venous catheter; HR: hazard ratio; ICU LOS: ICU length of hospital stay.

$P<0.001$ ), with an excess of US $\$ 18,854$ (in SICU) and US\$23,310 (total hospitalization) (Table 6).

\section{Cost-saving by intervention}

The number of MRSA cases prevented by the intervention was estimated to be 13 (6,817 patient days (period

Table 6 Comparison of hospital stay and hospital cost for patients with or without MRSA infections

\begin{tabular}{llll}
\hline Category & $\begin{array}{l}\text { Cases } \\
\text { with MRSA } \\
\text { infections }\end{array}$ & $\begin{array}{l}\text { Cases } \\
\text { without MRSA } \\
\text { infections }\end{array}$ & P value \\
\hline $\begin{array}{l}\text { Length of stay (days) } \\
\text { SICU length of stay (mean) }\end{array}$ & 40.6 & 6.6 & $<0.001$ \\
SICU length of stay (median) & 37 & 2 & $<0.001$ \\
$\begin{array}{l}\text { Total length of hospital } \\
\text { stay (mean) }\end{array}$ & 75.4 & 23.3 & $<0.001$ \\
$\begin{array}{l}\text { Total length of hospital } \\
\text { stay (median) }\end{array}$ & 59 & 15 & $<0.001$ \\
$\begin{array}{l}\text { Cost }{ }^{\text {a,b }} \\
\text { SICU costs (mean) }\end{array}$ & 25,466 & 6,612 & $<0.001$ \\
$\begin{array}{l}\text { SICU costs (median) } \\
\begin{array}{l}\text { Total hospitalization } \\
\text { costs (mean) }\end{array}\end{array}$ & 25,161 & 3,748 & $<0.001$ \\
$\begin{array}{l}\text { Total hospitalization } \\
\text { costs (median) }\end{array}$ & 31,815 & 8,505 & $<0.001$ \\
\hline
\end{tabular}

${ }^{\mathrm{a}}$ in US dollars (exchange ratio US\$1 $=\mathrm{NT} \$ 30$ ). ${ }^{\mathrm{b}}$ The medical cost, including physician care, accommodation, nursing care, meals, laboratory procedures, treatments, operations, rehabilitation programs, medications, pharmacy service and anesthesia. MRSA: methicillin-resistant Staphylococcus aureus; SICU: surgical intensive care unit.
2) $\times 0.00029$ (observed MRSA infection rate in period 2)/0.3 (adjusted HR of intervention) $+16,523$ patient days (period 4) $\times 0.00024$ (observed MRSA infection rate in period 4)/0.3 (adjusted HR of intervention)).

The cost saved by preventing 13 MRSA cases during the total 20-month intervention period (periods 2 and 4) was $13 \times$ US $\$ 23,310=$ US\$303,030 (annual cost saving: US\$190,908). The active surveillance and decolonization program cost US\$13,717 during the total 20-month intervention period (annual cost: US\$8,231). The costsaving ratio was 22 (US\$303,030/US $\$ 13,717$ ); thus, every dollar spent on the intervention resulted in a saving of $\$ 22$ in medical costs.

\section{Discussion}

Our results show that the active screening and decolonization program was associated with a decrease in all-type clinical in-hospital MRSA infections (adjusted OR: 0.3 ; $95 \%$ CI: 0.1 to 0.8 ) and a lower 90 -day mortality (adjusted HR: 0.8; 95\% CI: 0.7 to 0.99). Furthermore, the active screening and decolonization program is costsaving; every dollar spent on interventions resulted in a saving of $\$ 22$ in medical costs.

Due to ethical considerations, we were unable to use randomized controlled experiments to evaluate the active screening and decolonization program. Nevertheless, the interrupted time-series study design, in conjunction with the use of regression models to control the effects of hospital-level and patient-level confounders, strengthens the causal inference. The rapid drop, resurgence and drop again in MRSA infection rates, following introduction, interruption and reintroduction of the interventions in temporal sequence, makes a strong case against alternative explanations such as a progressive decline in MRSA carrier rates in community, or a continuing improvement in overall hospital infection control measures. Furthermore, after adjusting for effects of hospital-level improvements in infection-control practices (hand hygiene and bundle care), as well as individual patient-level risk factors (invasive procedures and severity of underlying diseases), the intervention remains an independent protective factor against MRSA infection and mortality.

The protective effect measured in our study includes both the direct effect and the indirect effect. Decolonization of MRSA carriers directly reduces his or her risk of subsequent MRSA infections. Moreover, decolonization of MRSA carriers prevents the transmission of MRSA to non-carriers that would otherwise happen, and therefore indirectly protects those who are not carriers at the time of ICU admission.

The impact of active screening and decolonization on MRSA transmission within hospitals is further highlighted by a decrease in the prevalence of MRSA carriers 
among hospitalized patients. Mathematical modeling studies predict that, in settings where MRSA carriage is endemic, the implementation of active screening and decolonization will lead to a rapid drop in MRSA carriage rate in hospitalized patients [22]. As predicted by modeling studies, the average MRSA-positive rate among surveillance cultures in our study rapidly dropped from the initial $11.3 \%$ (24 out of 213; period 2) to $6.1 \%$ (33 out of 538; period 4) $(P=0.038)$. The $47 \%$ decrease in MRSA carriage rate (from 11.3 to $6.1 \%$ ) within a short time period is consistent with the effect of the active screening and decolonization program in period 2 on blocking nosocomial MRSA transmission [22].

The use of intranasal mupirocin and chlorhexidine baths for decolonization in our study is likely the key factor of the observed efficacy. Two recent clusterrandomized trials conclusively showed that active surveillance and isolation alone, in the absence of a decolonization program, did not reduce the MRSApositive clinical culture rate or the MRSA bloodstream infection rate $[13,23,24]$. Although another study reported that universal surveillance, contact precaution and hand hygiene without decolonization were associated with a $62 \%$ decrease in MRSA infections in ICUs [25], an independent analysis of the data using mathematical modeling showed that only a very small fraction of the observed effect could be attributed to active detection and isolation alone [26].

MRSA infections increase the mortality of hospitalized patients by 12.4 to $28.5 \%$ [5]. Theoretically, prevention of HA-MRSA infections in ICU patients should lead to a reduction in mortality. However, the survival of ICU patients is heavily influenced by their acute severity of illnesses, prior length of stay and underlying diseases $[19,27]$, which need to be taken into account in analyzing the effect of interventions on the mortality of ICU patients. We showed that, after adjusting for effects of the above-stated variables, the active screening and decolonization program is an independent protective factor against mortality. Thus, decolonization of MRSA is not only an infection-control measure, but also could be a potentially life-saving intervention for all ICU patients in settings with a high MRSA infection rate.

In the present study, we used inexpensive conventional screening plates to detect MRSA carriers, with a turnaround time of two to three days. The efficacy of interventions may be better if rapid tests were used for screening to minimize the delay in initiating decolonization. Tests based on real-time PCR have a turnaround time of less than one day, but are expensive [28]. Culture-based methods using chromogenic screening media can yield a rapid result after 18 to 24 hours incubation, but sensitivity varies by product, and is usually lower than that of PCR-based methods [29].
Because of logistic consideration, we did not obtain extra-nasal cultures when screening individuals for MRSA colonization. Use of nasal culture to guide decolonization in our study, however, appears to be sufficient to yield a significant decrease in MRSA infection rates and decrease in mortality. The additional use of extra-nasal cultures will likely detect more MRSA carriers, and thus increase the impact of the intervention [30].

Cost is an important concern for the sustained implementation of HAI prevention efforts. Consistent with previous cost analyses of MRSA prevention programs [31-33], our data show that, because of the high excess cost associated with MRSA infection and related complications (US\$23,310 per MRSA infection case) in the SICU setting, the investment in active screening and decolonization (US\$8,231 per year) will actually be costsaving if at least one case of MRSA infection is prevented in the SICU every year. We estimated that 13 cases of MRSA infections had been prevented during the total 20-month intervention period, which yielded a highly beneficial cost-saving ratio of 22 .

An alternative to the screening followed by targeted decolonization approach is universal decolonization for all ICU patients [12,34]. A multicenter cluster-randomized trial showed that daily chlorhexidine baths for all ICU patients reduced hospital-acquired bloodstream infection rates by $28 \%$ [34]. The REDUCE MRSA trial further demonstrated that universal administration of intranasal mupirocin and daily chlorhexidine baths in ICUs was more effective at reducing the MRSA clinical culture rate and bloodstream infection rate from any pathogen than was targeted decolonization [12]. Universal decolonization eliminates the problem of false negative screening results. There will be no delay in initiating decolonization [12]. Additional advantages include reduction in contact precaution, as well as reduction in infections caused by bacteria other than MRSA [12]. It is reasonable to expect that universal decolonization will have a greater effect in reducing mortality of ICU patients than targeted decolonization. However, an important concern for the universal use of mupirocin for all ICU patients is selection for mupirocin-resistant strains [24,35]. Moreover, for those ICU patients who do not carry MRSA, the use of mupirocin is arguably not justified. Until these concerns can be adequately addressed, targeted decolonization remains an important option for reducing MRSA-related morbidity and mortality in routine clinical settings.

Our study has several limitations. First, our results in the SICU may not be generalizable to medical ICUs, where patients generally have prolonged hospital stays and more complicated illnesses than patients in SICUs. Second, we did not conduct a molecular analysis of the MRSA strains isolated during the study periods to differentiate MRSA of endogenous origin from MRSA that 
was exogenously acquired through intra-hospital transmission. Additional limitations included the lack of follow-up cultures during the study period; thus, the effect of the mupirocin eradication of the MRSA in the carriers was not documented. Further monitoring of MRSA susceptibility to mupirocin is necessary to ensure the long-term efficacy of the program.

\section{Conclusions}

Active screening for MRSA and decolonization in ICU settings with a high MRSA infection rate is associated with a decrease in MRSA infections, mortality and medical cost.

\section{Key messages}

- Methicillin-resistant Staphylococcus aureus (MRSA) is a leading pathogen of healthcare-associated infections in intensive care units (ICUs).

- Routine active screening for MRSA and decolonization in ICU settings is associated with a decrease in MRSA infections, mortality and medical cost in settings with a high MRSA infection rate.

- Both MRSA carriers and non-carriers can benefit from a routine active MRSA screening and decolonization program.

- In settings where MRSA is endemic, MRSA carriage rate drops after implementation of the active screening and decolonization program.

\section{Additional file}

Additional file 1: IRB certificate for approval.

\section{Abbreviations}

APACHE II: Acute Physiology and Chronic Health Evaluation II;

HA: Hospital-acquired; HAl: Healthcare-associated infection; HA-MRSA: Healthcare-associated methicillin-resistant Staphylococcus aureus; HR: Hazard ratio; ICU: Intensive care unit; IRB: Institutional review board; MRSA: Methicillin-resistant Staphylococcus aureus; OR: Odds ratio; SICU: Surgical intensive care unit; TMUH: Taipei Medical University Hospital; VAP: Ventilator-associated pneumonia.

\section{Competing interests}

The authors declare that they have no competing interests.

\section{Authors' contributions}

YJL and CTF conceived of and designed the study. YJL, JZC, HCL, HYL, SYL and PRH acquired the data. JZC, CTF, and HHL analyzed data and interpreted results. JZC and CTF drafted the manuscript. CTF, HHL, SYL, and PRH critically revised the manuscript for important intellectual content. YJL and HYL obtained funding. YJL, HCL, CTF, and PRH provided administrative, technical or material support. YJL, CTF, HCL, and PRH supervised the study. All authors read and approved the final manuscript and agree to be accountable for all aspects of the work.

\section{Acknowledgements}

This study was supported by the Taipei Medical University Hospital, Taipei, Taiwan (grant number GH8106).

\section{Author details}

'Division of Infectious Diseases, Department of Internal Medicine, Taipei Medical University Hospital, 252 Wusing Street, Taipei 11031, Taiwan. ${ }^{2}$ Graduate Institute of Clinical Medicine, College of Medicine, Taipei Medical University, 250 Wusing Street, Taipei 11031, Taiwan. ${ }^{3}$ Department of Infection Control, Taipei Medical University Hospital, 252 Wusing Street, Taipei 11031, Taiwan. ${ }^{4}$ Department of Pediatrics, School of Medicine, College of Medicine, Taipei Medical University, 250 Wusing Street, Taipei 11031, Taiwan. ${ }^{5}$ Department of Laboratory Medicine, Taipei Medical University Hospital, 252 Wusing Street, Taipei 11031, Taiwan. ${ }^{6}$ Department of General Medicine, School of Medicine, College of Medicine, Taipei Medical University, 250 Wusing Street, Taipei 11031, Taiwan. ${ }^{7}$ Institute of Epidemiology and Preventive Medicine, College of Public Health, National Taiwan University, 17 Xu-Zhou Road, Taipei 10002, Taiwan. ${ }^{8}$ Department of Internal Medicine, National Taiwan University Hospital, 7 Chun-Shan South Road, Taipei 10002, Taiwan. ${ }^{9}$ Department of Laboratory Medicine, National Taiwan University Hospital, National Taiwan University College of Medicine, 1 Jen-Ai Road, Taipei 10055, Taiwan.

Received: 30 January 2015 Accepted: 11 March 2015

Published online: 08 April 2015

\section{References}

1. Jevons MP, Coe AW, Parker MT. Methicillin resistance in staphylococci. Lancet. 1963;1:904-7.

2. Huang SS, Yokoe DS, Hinrichsen VL, Spurchise LS, Datta R, Miroshnik I, et al. Impact of routine intensive care unit surveillance cultures and resultant barrier precautions on hospital-wide methicillin-resistant Staphylococcus aureus bacteremia. Clin Infect Dis. 2006;43:971-8.

3. Tenover FC, Biddle JW, Lancaster MV. Increasing resistance to vancomycin and other glycopeptides in Staphylococcus aureus. Emerg Infect Dis. 2001;7:327-32.

4. Hsueh PR, Lee SY, Perng CL, Chang TY, Lu JJ. Clonal dissemination of meticillin-resistant and vancomycin-intermediate Staphylococcus aureus in a Taiwanese hospital. Int J Antimicrob Agents. 2010;36:307-12.

5. Su CH, Chang SC, Yan JJ, Tseng SH, Chien LJ, Fang CT. Excess mortality and long-term disability from healthcare-associated Staphylococcus aureus infections: a population-based matched cohort study. PLoS One. 2013;8, e71055.

6. von Eiff C, Becker K, Machka K, Stammer H, Peters G. Nasal carriage as a source of Staphylococcus aureus bacteremia. N Engl J Med. 2001;344:11-6.

7. Wertheim HFL, Melles DC, Vos MC, Leeuwen WV, Belkum AV, Verbrugh HA, et al. The role of nasal carriage in Staphylococcus aureus infections. Lancet Infect Dis. 2005;5:751-62.

8. Sandri AM, Dalarosa MG, Alcantara LR, Silva Elias L, Zavascki AP. Reduction in incidence of nosocomial methicillin-resistant Staphylococcus aureus (MRSA) infection in an intensive care unit: role of treatment with mupirocin ointment and chlorhexidine baths for nasal carriers of MRSA. Infect Control Hosp Epidemiol. 2006;27:185-7.

9. Ridenour G, Lampen R, Federspiel J, Kritchevsky S, Wong E, Climo M. Selective use of intranasal mupirocin and chlorhexidine bathing and the incidence of methicillin-resistant Staphylococcus aureus colonization and infection among intensive care unit patients. Infect Control Hosp Epidemiol. 2007;28:1155-61.

10. Fraser TG, Fatica C, Scarpelli M, Arroliga AC, Guzman J, Shrestha NK, et al. Decrease in Staphylococcus aureus colonization and hospital-acquired infection in a medical intensive care unit after institution of an active surveillance and decolonization program. Infect Control Hosp Epidemiol. 2010;31:779-83.

11. Milstone AM, Budd A, Shepard JW, Ross T, Aucott S, Carroll KC, et al. Role of decolonization in a comprehensive strategy to reduce methicillin-resistant Staphylococcus aureus infections in the neonatal intensive care unit: an observational cohort. Infect Control Hosp Epidemiol. 2010;31:558-60.

12. Huang YC, Lien Rl, Su LH, Chou YH, Lin TY. Successful control of methicillinresistant Staphylococcus aureus in endemic neonatal intensive care units-a 7-year campaign. PLoS One. 2011;6, e23001.

13. Huang SS, Septimus E, Kleinman K, Moody J, Hickok J, Avery TR, et al. Targeted versus universal decolonization to prevent ICU infection. N Engl J Med. 2013;368:2255-65.

14. McGinigle KL, Gourlay ML, Buchanan IB. The use of active surveillance cultures in adult intensive care units to reduce methicillin-resistant Staphylococcus aureus-related morbidity, mortality, and costs: a systematic review. Clin Infect Dis. 2008;46:1717-25.

15. Glick SB, Samson DJ, Huang E, Vats V, Weber S, Aronson N, et al. Screening for methicillin-resistant Staphylococcus aureus (MRSA). Rockville (MD): Agency for Healthcare Research and Quality (US); 2013 Jun. Report No.: 13-EHC043-EF. 
16. Chang SC, Hsu LY, Luh KT, Hsieh WC. Methicillin-resistant Staphylococcus aureus infection. J Formos Med Assoc. 1988;87:157-63.

17. Chang SC, Wang YC, Chou WH, Sheng WH, Wang LS, Wang JT, et al. Taiwan Nosocomial Surveillance System (TNIS) 2011 Annual Surveillance Report. Infect Control J. 2012;22:301-7.

18. Harris AD, McGregor JC, Perencevich EN, Furuno JP, Zhu J, Peterson D, et al. The use and interpretation of quasi-experimental studies in medical informatics. J Am Med Inform Assoc. 2006;13:16-23.

19. Fang CT, Shau WY, Chen YC, Chen YC, Wang JT, Hung CC, et al. Early empirical glycopeptide therapy for patients with methicillin-resistant Staphylococcus aureus bacteraemia: impact on the outcome. J Antimicrob Chemother. 2006;57:511-9.

20. Horan TC, Andrus M, Dudeck MA. CDC/NHSN surveillance definition of health care-associated infection and criteria for specific types of infections in the acute care setting. Am J Infect Control. 2008:36:309-32.

21. Garner JS, Jarvis WR, Emori TG, Horan TC, Hughes JM. CDC definitions for nosocomial infections, 1988. Am J Infect Control. 1988;16:128-40.

22. Gurieva TV, Bootsma MC, Bonten MJ. Decolonization of patients and health care workers to control nosocomial spread of methicillin-resistant Staphylococcus aureus: a simulation study. BMC Infect Dis. 2012;12:302.

23. Huskins WC, Huckabee CM, O'Grady NP, Murray P, Kopetskie H, Zimmer L, et al. Intervention to reduce transmission of resistant bacteria in intensive care. N Engl J Med. 2011;364:1407-18.

24. Edmond MB, Wenzel RP. Screening inpatients for MRSA - case closed. N Engl J Med. 2013;368:2314-5.

25. Jain R, Kralovic SM, Evans ME, Ambrose M, Simbartl LA, Obrosky DS, et al. Veterans Affairs Initiative to prevent methicillin- resistant Staphylococcus aureus infections. N Engl J Med. 2011:364:1419-30.

26. Gurieva T, Bootsma MCJ, Bonten MJM. Successful Veterans Affairs Initiative to prevent methicillin-resistant Staphylococcus aureus infections revisited. Clin Infect Dis. 2012;54:1618-20.

27. Johnson AE, Kramer AA, Clifford GD. A new severity of illness scale using a subset of Acute Physiology And Chronic Health Evaluation data elements shows comparable predictive accuracy. Crit Care Med. 2013;41:1711-8.

28. Roisin S, Laurent C, Denis O, Dramaix M, Nonhoff C, Hallin M, et al. Impact of rapid molecular screening at hospital admission on nosocomial transmission of methicillin-resistant Staphylococcus aureus: cluster randomised trial. PLoS One. 2014;9:e96310.

29. Luteijn JM, Hubben GA, Pechlivanoglou P, Bonten MJ, Postma MJ. Diagnostic accuracy of culture-based and PCR-based detection tests for methicillin-resistant Staphylococcus aureus: a meta-analysis. Clin Microbiol Infect. 2011;17:146-54

30. Bode LG, Kluytmans JA, Wertheim HF, Bogaers D, Vandenbroucke-Grauls $C M$, Roosendaal $R$, et al. Preventing surgical-site infections in nasal carriers of Staphylococcus aureus. N Engl J Med. 2010;362:9-17.

31. Wernitz MH, Keck S, Swidsinski S, Schulz S, Veit SK. Cost analysis of a hospital-wide selective screening programme for methicillin-resistant Staphylococcus aureus (MRSA) carriers in the context of diagnosis related groups (DRG) payment. Clin Microbiol Infect. 2005:11:466-71.

32. Gavalda L, Masuet C, Beltran J, Garcia M, Garcia D, Sirvent JM, et al. Comparative cost of selective screening to prevent transmission of methicillinresistant Staphylococcus aureus (MRSA), compared with the attributable costs of MRSA infection. Infect Control Hosp Epidemiol. 2006;27:1264-6.

33. Sheng WH, Wang JT, Lu DCT, Chie WC, Chen YC, Chang SC. Comparative impact of hospital-acquired infections on medical costs, length of hospital stay and outcome between community hospitals and medical centres. J Hosp Infect. 2005;59:205-14.

34. Climo MW, Yokoe DS, Warren DK, Perl TM, Bolon M, Herwaldt LA, et al Effect of daily chlorhexidine bathing on hospital-acquired infection. N Engl J Med. 2013:368:533-42.

35. Patel JB, Gorwitz RJ, Jernigan JA. Mupirocin resistance. Clin Infect Dis. 2009:49:935-41.

\section{Submit your next manuscript to BioMed Central and take full advantage of:}

- Convenient online submission

- Thorough peer review

- No space constraints or color figure charges

- Immediate publication on acceptance

- Inclusion in PubMed, CAS, Scopus and Google Scholar

- Research which is freely available for redistribution 